\title{
Strikingly different luminescent properties arising from single crystals grown from solution or from the vapor phase in a diketo-pyrrolo-pyrrole analog
}

\author{
Tomohiko Imoda and Jin Mizuguchi ${ }^{\text {a) }}$ \\ Department of Applied Physics, Graduate School of Engineering, Yokohama National University, \\ 240-8501 Yokohama, Japan
}

(Received 26 February 2007; accepted 30 July 2007; published online 12 October 2007)

\begin{abstract}
The title compound di-cyano-pyrrolo-pyrrole (DCPP) is an analog of diketo-pyrrolo-pyrrole (DPP) known as a red pigment on the market. Brilliant orange photoluminescence has been observed in crystals of DCPP recrystallized from solution (crystal I); whereas the luminescence was quite weak in single crystals grown from the vapor phase (crystal II). To elucidate the difference in luminescent properties, a series of structural analysis together with thermal analysis has been carried out on crystals I and II at $-180{ }^{\circ} \mathrm{C}$, RT, $150{ }^{\circ} \mathrm{C}$, and $200{ }^{\circ} \mathrm{C}$. However, no difference in structure is recognized between crystals I and II within the precision of the X-ray analysis. Nevertheless, a slight difference in sublimation temperature of about $5^{\circ}$ is observed between crystals I and II. Since the sublimation temperature depends on the cohesion in the solid state and the cohesion, in turn, governs the extent of lattice vibration (i.e., nonradiative process), a slight difference in sublimation temperature appears to determine the fraction between the radiative and nonradiative processes in crystals I and II. C 2007 American Institute of Physics. [DOI: 10.1063/1.2784996]
\end{abstract}

\section{INTRODUCTION}

Electroluminescence (EL) has been the focus of considerable interest because of its high potential for compact and clear display devices. ${ }^{1}$ Although a great number of compounds are known which fluoresce in solution, the fluorophores in the solid state are relatively limited. In the course of our studies on the electronic structure of organic pigments, ${ }^{2}$ we encountered a compound [di-cyano-pyrrolopyrrole (DCPP) shown in Fig. 1(a)] (i.e., a derivative of well-known diketo-pyrrolo-pyrroles (DPPs) ${ }^{3-5}$ [DPPs: Fig. 1(b)] that emits intense orange photoluminescence in the solid state. However, this is only specific of crystals grown from solution (crystal I), not of the crystal grown from the vapor phase (crystal II). Furthermore, the present compound exhibits no photoluminescence in solution. This evidently indicates that the emission in the solid state involves intermolecular interactions. Only a few compounds are known which possess the above luminescent property, such as tris $(8-$ hydroxyquinolino)aluminum $\left(\mathrm{Alq}_{3}\right){ }^{6}{ }^{6} \alpha$-pyrone, ${ }^{7} \quad$ and oligopyridine. ${ }^{8}$

At first, we firmly believed that the crystal structure of crystals I and II must be different and started our investigation by structural analysis on single crystals. It turned out, however, that the structure was exactly the same within the precision of the X-ray structure analysis. Since, in inorganic phosphors, luminescence intensity is known to greatly depend on the purity and imperfections of host crystals, although their crystal structure is the same, and even a trace impurity can quench totally the solid-state luminescence. Therefore, special attention has been paid in the present investigation to the purification of DCPP. Attention was also focused on the preparation of single crystals from various solutions, or from the vapor phase. Then, a series of structural analysis was carried out on crystals I and II of DCPP at temperatures of $-180{ }^{\circ} \mathrm{C}, \mathrm{RT},{ }^{9} 150{ }^{\circ} \mathrm{C}$, and $200{ }^{\circ} \mathrm{C}$. In spite of these careful experiments, no noticeable difference in structure was recognized between crystals I and II. However, a slight difference was observed in sublimation temperature, which is closely correlated with the lattice vibration.

The present paper discusses the correlation between the difference in luminescent properties and the difference in sublimation temperature in crystals I and II on the basis of the crystal structure and thermal analysis.

\section{EXPERIMENT}

\section{A. Sample preparation, purification, and crystal growth}

DCPP was prepared in two steps through 2,5-dimethyl DPP as shown in Scheme 1. 2,5-Dimethyl DPP was obtained by reaction of DPP with methyl $p$-toluenesulfonate in nitrobenzene in the presence of $\mathrm{K}_{2} \mathrm{CO}_{3}$ at $200-225{ }^{\circ} \mathrm{C}$ for 1 h. ${ }^{10}$ Then, DCPP was prepared by reacting 2,5-dimethyl DPP with bis(trimethylsilyl)carbodiimide in the presence of tita- (a)

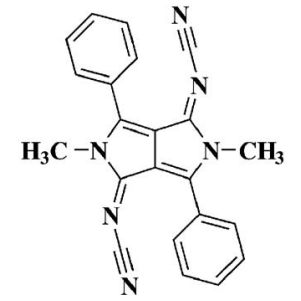

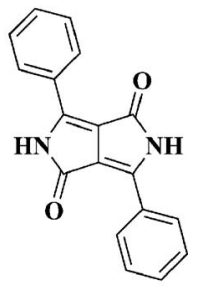

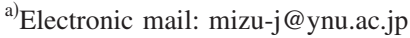

FIG. 1. Molecular conformation: (a) DCPP and (b) DPP. 


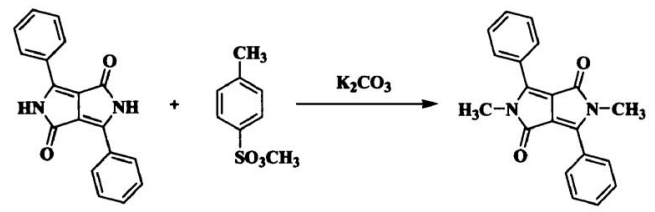

II

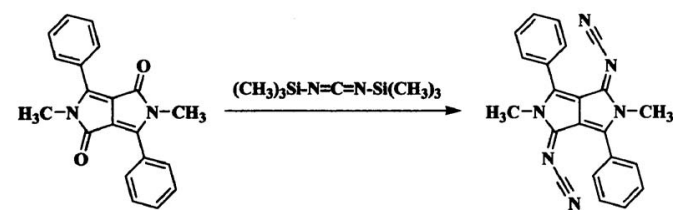

SCHEME 1. Two-step reaction for the synthesis of DCPP.

nium tetrachloride at $80{ }^{\circ} \mathrm{C}$ for 8 days. ${ }^{11}$ After that, the product was purified five times by sublimation at about $340^{\circ} \mathrm{C}$, using a two-zone furnace. ${ }^{12}$

Single crystals of DCPP were grown by recrystallization from various solutions in methylene chloride, acetone, acetonitrile, and dimethylsulfoxide. A number of single crystals of orange color (crystal I) were always isolated in the form of thin platelets, or scalelike crystals, quite irrespective of solvents used. Crystal I fluoresces intensely as recognized at first sight at RT. The orange color is attributed to both the optical absorption and luminescence of crystal I.

On the other hand, single crystals were also grown from the vapor phase in a closed system with a sublimation temperature of about $300{ }^{\circ} \mathrm{C}$ for $48 \mathrm{~h}$. Prismatic crystals of dark red color (crystal II) were obtained in the low-temperature region of about $200{ }^{\circ} \mathrm{C}$. However, crystal II exhibits quite weak luminescence, peaking at a slightly shorter wavelength than that of crystal I. The dark red color is due primarily to the optical absorption of the material and secondarily to the weak luminescence.

\section{B. Interconversion test between crystals I and II}

Since a negligibly small amount of impurity is known to quench the solid-state luminescence, the following interconversion test was carried out with great care in order to ensure that the difference in luminescence between crystals I and II arises from their intrinsic properties. The results were fully reproducible. The DCPP crystals which were recrystallized from solution in methylene chloride exhibit brilliant orange luminescence. In addition, the crystals grown from different solvents such as acetone, acetonitrile, and dimethylsulfoxide (DMSO) are found to behave exactly in the same way. Next, the vapor growth was made in a closed system by using the above single crystals grown from solution, for example, methylene chloride. We obtained a number of prismatic crystals of the dark red color, characterized by weak luminescence. Then, these single crystals were dissolved again, for example, in methylene chloride and then recrystallized. This gave again platelet crystals with intense orange luminescence. The above interconversion was repeated three times, ending up always with the same result. Likewise, the single crystals grown from other solvents (acetone, acetonitrile, and DMSO) were also tested to give the same result.
TABLE I. MO calculations for DCPP.

\begin{tabular}{llll}
\hline & $\begin{array}{c}\text { Heat of formation } \\
(\mathrm{kJ} / \mathrm{mol})\end{array}$ & $\lambda(\mathrm{nm})$ & $f$ (Oscillator strength) \\
Form I & Absorption band & 0.69 \\
Form II & & \\
\hline
\end{tabular}

\section{Equipment and measurements}

UV-visible (UV-vis) absorption and fluorescent spectra were recorded on a UV-2400PC spectrophotometer and a RF-5300PC spectrofluorophotometer, respectively, both of which are from Shimadzu. Temperature dependence of the photoluminescence was measured at $-50{ }^{\circ} \mathrm{C}$, RT, $150{ }^{\circ} \mathrm{C}$, and $250{ }^{\circ} \mathrm{C}$. Structural analysis was carried out at $-180^{\circ} \mathrm{C}$, RT, $150{ }^{\circ} \mathrm{C}$, and $200{ }^{\circ} \mathrm{C}$, using a Rigaku diffractometer of RAPID F for data collection. Measurements were made on one single sample for crystal I or crystal II, starting from low to high temperatures. The structure was solved by SHELXS $86^{13}$ and refined by TEXSAN. ${ }^{14}$

Thermogravimetric analysis and differential thermal analysis (TGA/DTA) as well as differential scanning calorimetry (DSC) measurements were carried out with great care at different heating rates with different sample weights, because the TGA/DTA and DSC curves are dependent on these parameters. TGA/DTA measurements were made on powdered samples in air or under vacuum by a Rigaku Thermo Plus TG-8120 at a heating rate of 5 or $10{ }^{\circ} \mathrm{C} / \mathrm{min}$ while the sample weight was 5 or $10 \mathrm{mg}$. Heat flow was also measured in air as a function of temperature by means of DSC (Thermo Plus DSC8230) from Rigaku. The heating rate was 5 or $10{ }^{\circ} \mathrm{C}$ per minute for the sample weight of 5 or 10 $\mathrm{mg}$.

\section{Molecular orbital (MO) calculations}

Two forms of the molecular configuration are possible, as shown below Table I, depending on the inner and outer position of the cyano group: form I and form II, respectively. The geometry of DCPP molecule was optimized by means of the AM1 Hamiltonian of MOPAC93. ${ }^{15}$ The spectroscopic calculations were then carried out on the optimized geometry using the INDO/S Hamiltonian in the ZINDO program package. $^{15}$

\section{RESULTS AND DISCUSSION}

\section{A. MO calculations}

Table I shows the heat of formation for forms I and II, together with their spectroscopic calculations. The heat of formation is higher in form II $(1096.3 \mathrm{~kJ} / \mathrm{mol})$ than in form I $(1055.6 \mathrm{~kJ} / \mathrm{mol})$. However, form II is actually the structure in the solid state, as shown below. In forms I and II, the 
TABLE II. Crystallographic parameters for crystals I and II at RT.

\begin{tabular}{ccc}
\hline \hline & Crystal I & Crystal II \\
\hline Crystal system & Monoclinic & Monoclinic \\
Space group & $P 2_{1} / c$ & $P 2_{1} / c$ \\
Molecular symmetry & $C_{i}$ & $C_{i}$ \\
$Z$ & 2 & 2 \\
$a(\AA)$ & $6.522(1)$ & $6.521(1)$ \\
$b(\AA)$ & $6.518(1)$ & $6.516(1)$ \\
$c(\AA)$ & $21.714(4)$ & $21.708(4)$ \\
$\beta\left({ }^{\circ}\right)$ & $91.91(1)$ & $91.98(1)$ \\
$V\left(\AA^{3}\right)$ & $922.5(3)$ & $921.9(10)$ \\
$d\left(\mathrm{~g} / \mathrm{cm}^{3}\right)$ & 1.31 & 1.31 \\
$R$ value & 0.046 & 0.051 \\
Size of the crystal $\left(\mathrm{mm}^{3}\right)$ & $1.40 \times 0.20 \times 0.07$ & $0.20 \times 0.07 \times 0.25$ \\
\hline \hline
\end{tabular}

longest-wavelength band is due to the HOMO/LUMO $\pi$ $-\pi^{*}$ electronic transition where HOMO and LUMO denote the highest occupied molecular orbital and the lowest unoccupied orbital, respectively. The absorption maximum of form II is positioned slightly at shorter wavelengths than that of form I.

\section{B. Crystal structure}

Table II details the crystallographic parameters at RT for crystal I grown from solution and crystal II grown from the vapor phase. Both crystals crystallize in the space group $P 2_{1} / c$ with a molecular symmetry of $C_{i}$. No noticeable difference in crystallographic parameters is recognized between crystals I and II within the precision of the x-ray analysis. Therefore, the structure is shown here only for crystal I at RT.

The ORTEP plot is shown in Fig. 2(a). The molecule possesses $C_{i}$. The phenyl rings are deviated, in the same

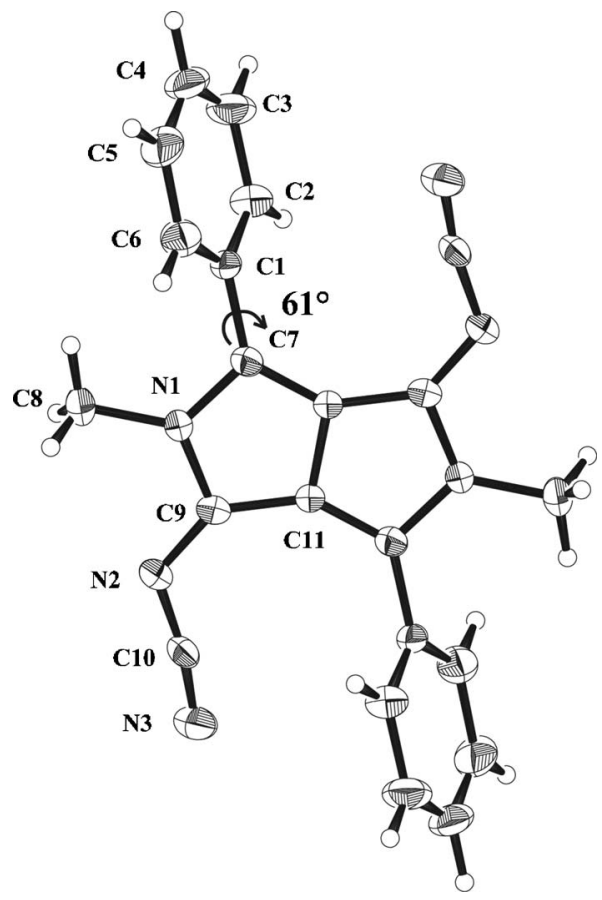

FIG. 2. ORTEP plot of DCPP. (a)
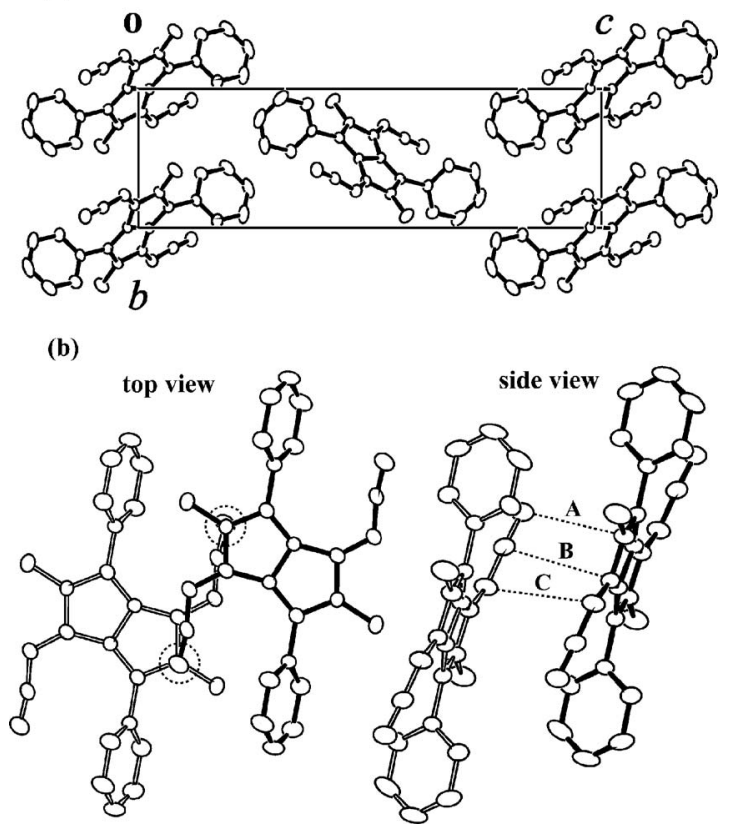

FIG. 3. (a) Projection of the crystal structure of DCPP onto the $(b, c)$ plane and (b) overlap of two molecules. A: $d(\mathrm{~N} 1 \cdots \mathrm{N} 3)=3.206 \AA$, B: $d(\mathrm{C} 9 \cdots \mathrm{C} 10)=3.337 \AA$, and $\mathrm{C}: d(\mathrm{~N} 2 \cdots \mathrm{N} 2)=3.625 \AA$.

direction, from the heterocyclic ring system by about $61^{\circ}$. The distance between N3 and C2 atoms corresponds approximately to that of the van der Waals radii. The angle of N2-C10-N3 atoms is not exactly $180^{\circ}$ because of the repulsion between N3 and C3 atoms. All other bond parameters of the present analog agree well with those of DPP. ${ }^{16,17}$ The present structure corresponds to form II, shown in the inset of Table I. Form II is less stable than form I in the free space, as judged from the heat of formation (Table I). Nevertheless, form II exists in the solid state, presumably because the higher packing (i.e., higher density) is possible with form II due to lesser steric hindrance as compared with that of form I.

Figure 3(a) shows the projection of the crystal structure onto the $(b, c)$. The molecules are arranged in a zigzag fashion along the $c$-axis. Figure 3(b) shows the overlap of two molecules along the $a$-axis: top view and side view. It is remarkable to note that the molecules are stacked with overlap occurring only at the cyano group (top view). N3 of the lower molecule is directly under $\mathrm{N} 1$ of the upper molecule. Likewise, N1 of the lower one is nearly under N3 of the upper one.

\section{Temperature dependence of the lattice parameters for crystals I and II}

Figures 4(a) and 4(b) show the temperature dependence of the lattice parameters together with that of the density for crystals I and II, respectively. The $R$ values for crystal I for $-180{ }^{\circ} \mathrm{C}$, RT, $150{ }^{\circ} \mathrm{C}$, and $200{ }^{\circ} \mathrm{C}$ were $0.061,0.046,0.059$, and 0.049 , respectively, whereas those for crystal II were $0.062,0.051,0.060$, and 0.055 . The value of the parameters is expressed in percentage relative to the value at $-180{ }^{\circ} \mathrm{C}$. The lattice constants $a, b$, and $c$ increase with increasing temperature, while the reverse is the case for angle $\beta$ and the 

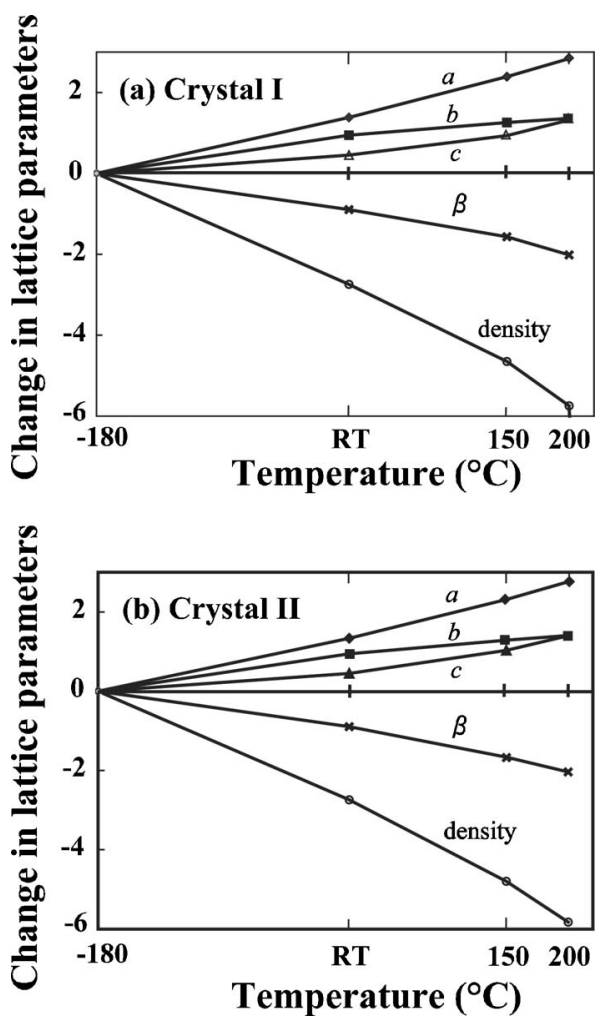

FIG. 4. Temperature dependence of the lattice parameters of DCPP at $-180^{\circ} \mathrm{C}$, RT, $150^{\circ} \mathrm{C}$, and $200{ }^{\circ} \mathrm{C}$ : (a) crystal I and (b) crystal II.

density. The value of the lattice $c$ coincides with that of lattice $b$ at $200{ }^{\circ} \mathrm{C}$. Exactly the same tendency is observed in crystal II.

\section{Solution spectra}

Figure 5 shows the solution spectra of DCPP in methylene chloride and acetonitrile. In the former spectrum, a prominent absorption band appears at around $518 \mathrm{~nm}$ together with two shoulders around 447 and $482 \mathrm{~nm}$, forming a progression of absorption bands. It is to be noted that the longest-wavelength band is quite steep and the absorption bands are equally spaced (about $1400 \mathrm{~cm}^{-1}$ ). Furthermore, the MO calculations predict only one electronic transition in the visible region (Table I). These facts suggest that the longest absorption band is assigned to the pure electronic transition (i.e., 0-0) and the second-longest wavelength band to the $0-1$, etc., as shown in Fig. 5, indicating that one single

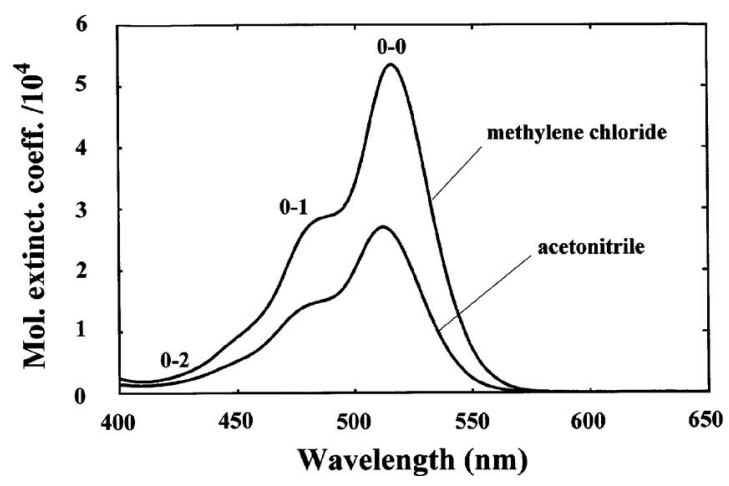

FIG. 5. Solution spectra of DCPP in methylene chloride and acetonitrile.
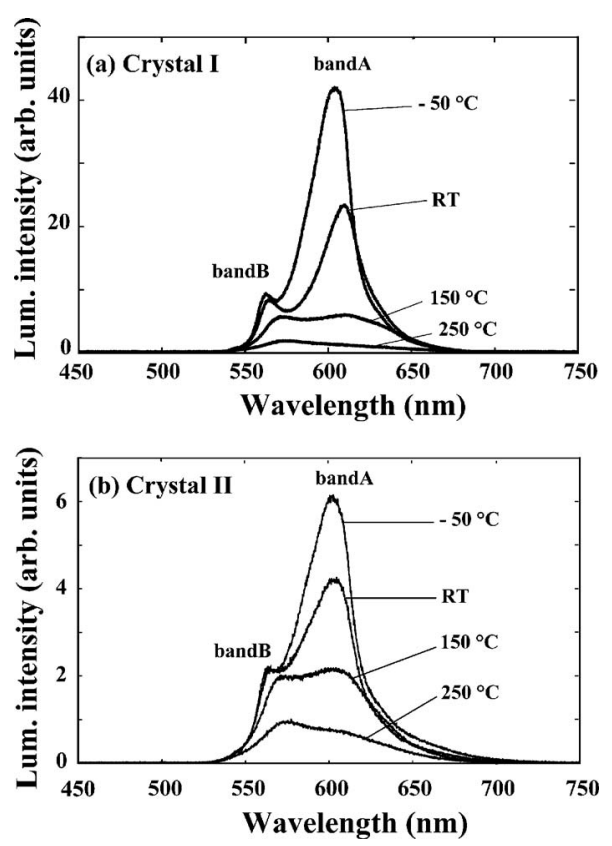

FIG. 6. Temperature dependence of the photoluminescence spectra of DCPP: (a) crystal I and (b) crystal II. The scale in intensity is in common in both figures.

electronic transition is coupled with vibrational transitions (i.e., vibronic transitions). The appearance of the progression of absorption bands is typical of the energy diagram where the potential minima of the ground and excited states occur at the same position of the coordinate.

\section{E. Temperature dependence of photoluminescence in crystals I and II}

Figures 6(a) and 6(b) show the temperature dependence of photoluminescence for crystals I and II, respectively, under the excitation of $365 \mathrm{~nm}$ measured at $-50{ }^{\circ} \mathrm{C}$, RT, $150{ }^{\circ} \mathrm{C}$, and $250{ }^{\circ} \mathrm{C}$. In crystal I, an intense band appears at $-50{ }^{\circ} \mathrm{C}$ around $605 \mathrm{~nm}$ (band $\mathrm{A}$ ), together with a shoulder around $564 \mathrm{~nm}$ (band $\mathrm{B}$ ). Band $\mathrm{A}$ is found to shift toward longer wavelengths as the temperature is increased from $-50{ }^{\circ} \mathrm{C}$ to RT. The intensity of the present luminescence decreases remarkably with increasing temperature and is completely quenched above $300{ }^{\circ} \mathrm{C}$ ("thermal quenching"). The spectral shape changes also drastically in such a way that band A decreases in intensity while band B remains nearly unaltered up to RT, accompanied by a slight spectral shift of both bands toward longer wavelengths. Then, band B is appreciably shifted to $571 \mathrm{~nm}$ at $150{ }^{\circ} \mathrm{C}$, whose intensity is now at the same level as that of band A. At $250{ }^{\circ} \mathrm{C}$, the intensity of band $\mathrm{B}$ is larger than that of band $\mathrm{A}$, although both intensities are quite weak. At $300{ }^{\circ} \mathrm{C}$, the photoluminescence is completely quenched. However, the luminescence reappears with decreasing temperature, indicating the reversibility of the appearance and disappearance of the photoluminescence.

The above results strongly suggest that the electronic structure of bands A and B is different, as will be discussed later. 

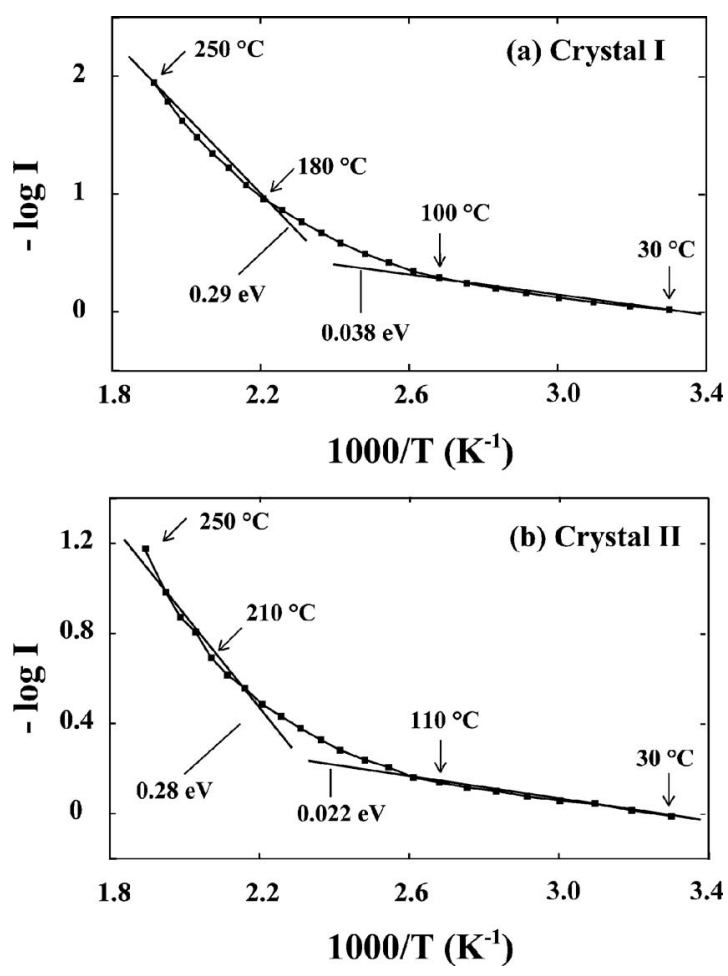

FIG. 7. Arrhenius plots of the integrated photoluminescence intensity (i) of DCPP: (a) crystal I and (b) crystal II. The luminescence quenches completely above $300{ }^{\circ} \mathrm{C}$ in both crystals.

In crystal II, the tendency of the temperature dependence is similar to that of crystal I, as shown in Fig. 6(b). However, the intensity is stronger in crystal I than in crystal II by a factor of 6-7 and that the peak shift in band A from $-50{ }^{\circ} \mathrm{C}$ to RT is very little as compared with that of crystal I. Crystal II is also found to exhibit thermal quenching at $300{ }^{\circ} \mathrm{C}$.

Figures 7(a) and 7(b) show the Arrhenius plot of the temperature dependence of the integrated fluorescence intensity for crystals I and II, respectively. Both temperature dependences are quite similar and are governed by double exponentials. The activation energy of the high-temperature region amounts to about $0.3 \mathrm{eV}$ in crystals I and II.

\section{F. Excitation spectra and the electronic structure in the solid state}

Excitation spectra were measured for crystals I and II by monitoring the fluorescence output at a certain wavelength while varying the wavelength of the exciting radiation. Such spectrum corresponds basically to the absorption spectrum of the crystal.

Figures 8(a) and 8(b) show the excitation spectra at RT for crystal I as monitored at 610 and $564 \mathrm{~nm}$ (bands A and B), respectively, together with the fluorescence spectrum of the solid state. It is remarkable to note that there are four bands in Fig. 8(a) in the solid state, one intense band at around $570 \mathrm{~nm}$ in addition to smaller bands or shoulders at around 540, 480, and $435 \mathrm{~nm}$, as indicated by arrows, whereas there exist only three bands in the molecular absorption spectrum in solution (Fig. 5). This indicates that one additional band appears as a result of intermolecular interactions on going from solution to the solid state. Since the band (a)

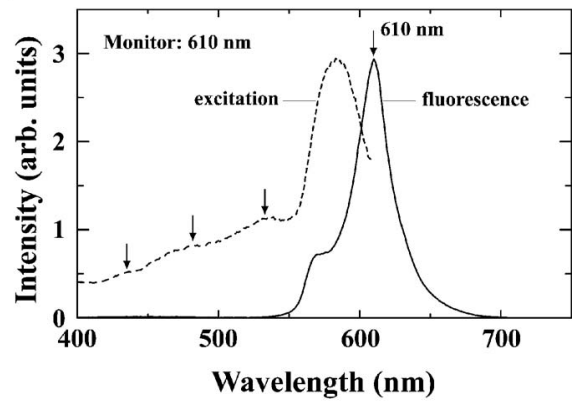

(b)

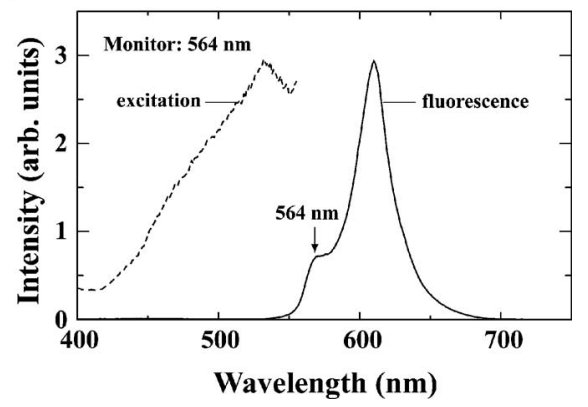

FIG. 8. Excitation and luminescence spectra for crystal I of DCPP: (a) monitored at $610 \mathrm{~nm}$ and (b) monitored at $564 \mathrm{~nm}$.

at around $570 \mathrm{~nm}$ is so different in intensity and also in spectral shape from the three bands at shorter wavelengths, it is highly probable that the band at around 570 is a new band caused by intermolecular interactions (for example, excitonic interactions), while the bands at shorter wavelengths are of the molecular nature: $0-0,0-1,0-2$ transitions in Fig. 5 . This suggests that emission band A is mainly caused by intermolecular interactions while emission band B is due to individual molecules. This agrees with the result of the temperature dependence of the photoluminescence (Fig. 6), which suggests that the electronic structure of bands A and B is different.

The excitation spectra for crystal II as monitored at 606 and $565 \mathrm{~nm}$ is given in Figs. 9(a) and 9(b), respectively. The spectral behavior is mostly similar to that of crystal I, although the bands are less structured. Particularly, no excitation maximum was observed in Fig. 9(b). It is also interesting to note that the luminescence peaks in both crystals are slightly different: 564 and $610 \mathrm{~nm}$ in crystal I (Fig. 8) while 565 and $606 \mathrm{~nm}$ in crystal II (Fig. 9).

\section{G. TGA/DTA and DSC measurements and the difference in luminescent properties}

Since TGA/DTA and DSC curves are dependent on the heating rate and sample weight, we have performed the analysis with different heating rates $\left(5\right.$ and $\left.10{ }^{\circ} \mathrm{C} / \mathrm{min}\right)$ and sample weights $(5$ and $10 \mathrm{mg}$ ) to confirm the reproducibility of the difference in sublimation temperature between crystals I and II. The sublimation temperature became slightly higher with the increase in heating rate, as observed in general. Although the values of the sublimation temperature were slightly different in all experiments, the reproducible results were always obtained that the sublimation temperature was 
(a)

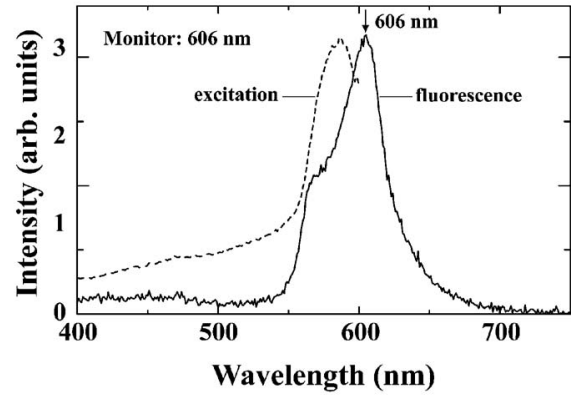

(b)

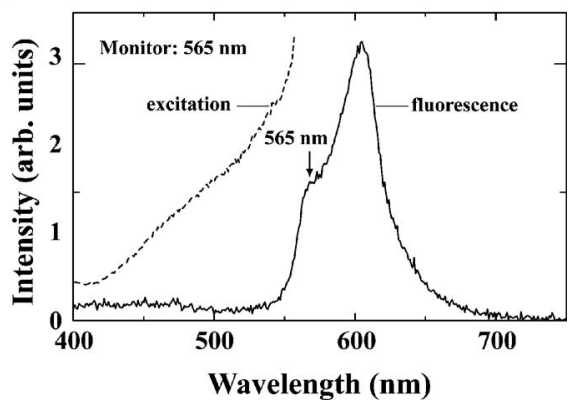

FIG. 9. Excitation and luminescence spectra for crystal II of DCPP: (a) monitored at $606 \mathrm{~nm}$ and (b) monitored at $565 \mathrm{~nm}$.

higher in crystal II than in crystal I by about $5{ }^{\circ} \mathrm{C}$. In addition, we have also carried out TGA/DTA measurements under vacuum. The following results are those with a heating rate of $5{ }^{\circ} \mathrm{C} / \mathrm{min}$ and a sample weight of $5 \mathrm{mg}$.

Figures 10(a) and 10(b) show the TGA/DTA curves for crystals I and II in air. The sublimation point is defined here as the DTA peak point, rather than the one as obtained by extrapolation of the TGA curve. The sublimation occurs at $369.4^{\circ} \mathrm{C}$ in crystal I while $373.5^{\circ} \mathrm{C}$ in crystal II. Under vacuum, on the other hand, the sublimation temperature was
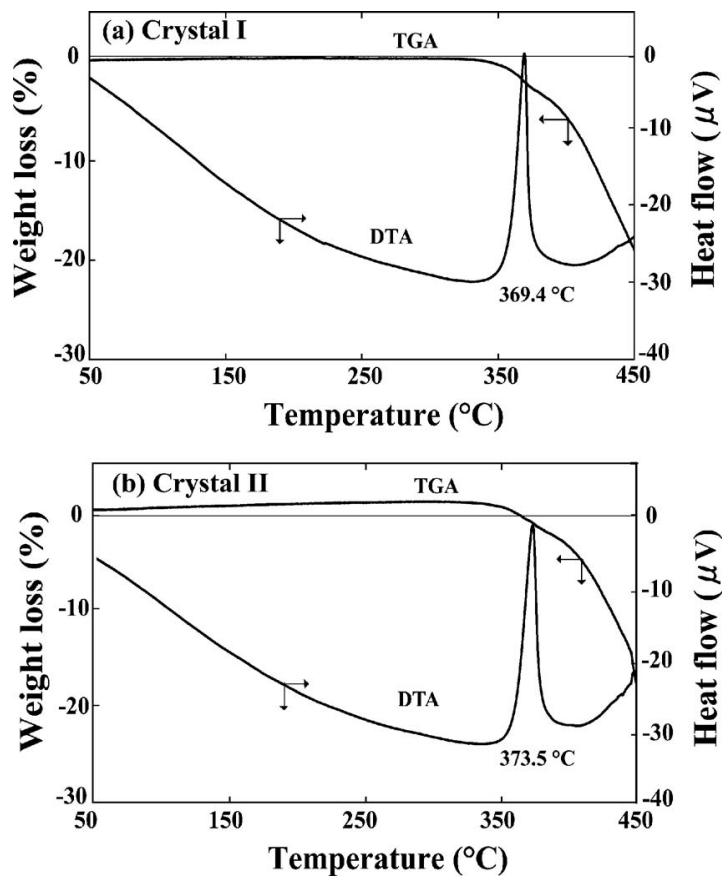

FIG. 10. TGA/DTA diagrams measured in air: (a) crystal I and (b) crystal II.
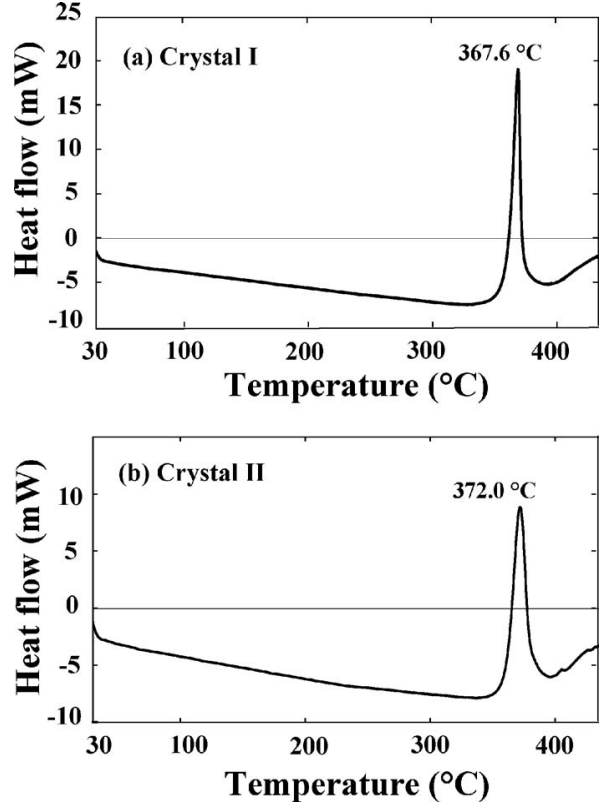

FIG. 11. DSC diagrams measured in air: (a) crystal I and (b) crystal II.

basically lower in both crystals I and II (not shown here) and the difference in sublimation temperature was slightly larger (i.e., $7.7^{\circ} \mathrm{C}$ ): 362.1 and $369.8^{\circ} \mathrm{C}$ for crystals I and II, respectively. This indicates that the cohesion in the solid state (which is directly correlated with the lattice energy) is larger in crystal I than that in crystal II. It is also noted that the weight loss in both crystals I and II proceed by two steps. This was also the case under vacuum (not shown here). The reason for the two-step process is not known for certain at the moment.

A distinct difference in DSC diagram in air is also observed between crystals I and II, as shown in Fig. 11, indicating that the sublimation takes place at 367.6 and $372.0^{\circ} \mathrm{C}$, respectively. Here again, crystal $\mathrm{I}$ is confirmed to sublime at a lower temperature than crystal II. The DSC peak of crystal II seems to be broader, which could be due to the more heterogeneous crystal structure.

\section{H. Correlation between lattice vibration and nonradiative process}

It is generally accepted that the sublimation temperature is correlated with the cohesion in the solid state. Since the cohesion in the solid state determines the extent of lattice vibration, higher sublimation temperature may result in an enhanced nonradiative decay process from the excited state through the lattice vibration. However, our observed difference in sublimation temperatures seems to be rather too small to apply this general rule directly, and further study is required to confirm this interpretation.

\section{Franck-Condon diagrams in solution and in the solid state}

We propose first the Franck-Condon configuration coordinate (FC CC) diagram for the solution on the basis of the observation of the progression of absorption bands (Fig. 5). The appearance of the progression suggests that the potential 
(a)

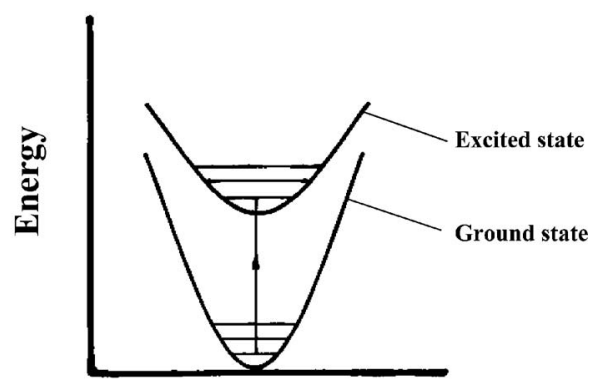

Configuration coordinate

(b)

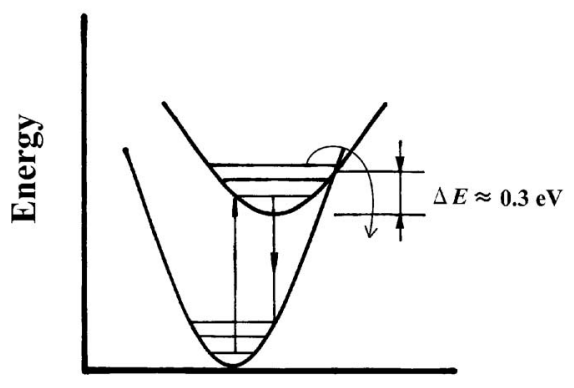

Configuration coordinate

FIG. 12. Proposed FC CC diagrams for DCPP: (a) solution and (b) crystals I and II.

minima of the ground and excited states are present at the same position of the configuration coordinate. This enables us to illustrate the FC CC diagram as shown in Fig. 12(a) for the solution state.

The diagram in the solid state is different from the solution one. Thermal quenching of fluorescence is observed in both crystals I and II. Thermal quenching is usually explained by means of the energy dissipation through the cross point of the ground state and of the excited state potentials. ${ }^{18}$ This activation process is governed by Boltzmann's distribution. The potential barrier which the electrons must surmount amounts to about $0.3 \mathrm{eV}$, as obtained by temperature dependence of the photoluminescence (Fig. 7). On the basis of the above consideration, we propose a FC CC diagram for crystals I and II, as shown in Fig. 12(b).

\section{CONCLUSIONS}

The unusual luminescent properties of two differently prepared DCPP crystals (crystals I and II) have been investigated from the standpoint of the crystal structure and thermal analysis. The following conclusions can be drawn from the present study:

1. DCPP exhibits no luminescence in solution while it fluoresces in the solid state. This indicates that the emission process involves intermolecular interactions.

2. Crystal I (recrystallized from solution) exhibits, by far, stronger luminescence than crystal II (grown from the vapor phase) by a factor of $6-7$, although no structural difference is recognized between them within the precision of the $\mathrm{x}$-ray analysis.

3. Both crystals I and II exhibit fluorescence quenching at about $300{ }^{\circ} \mathrm{C}$.

4. A slight difference in sublimation temperature was recognized between crystals I and II. This is presumably correlated with the difference in lattice vibration and is considered to determine the fraction between the radiative and nonradiative processes in crystals I and II.

${ }^{1}$ C. W. Tang and S. A. Van Slyke, Appl. Phys. Lett. 51, 913 (1987).

${ }^{2}$ W. Herbst and K. Hunger, Industrial Organic Pigments: Production, Properties, Applications, 3rd ed. (VCH, Weinheim, 2004).

${ }^{3}$ J. Mizuguchi, J. Phys. Chem. A 104, 1817 (2000).

${ }^{4}$ H. Takahashi and J. Mizuguchi, J. Electrochem. Soc. 152, H69 (2005).

${ }^{5}$ J. Mizuguchi, T. Imoda, H. Takahashi, and H. Yamakami, Dyes Pigments 68, 47 (2006).

${ }^{6}$ K. Araki, K. Mutai, Y. Shigematsu, M. Yamada, T. Nakajima, S. Kuroda, and I. Shimano J. Chem. Soc. [Perkin 1] 2, 613 (1996).

${ }^{7}$ K. Hirano, S. Minakata, M. Komatsu, and J. Mizuguchi, J. Phys. Chem. A 106, 4868 (2002).

${ }^{8}$ T. Mutai, H. Satou, and K. Araki, Nat. Mater. 4, 685 (2005).

${ }^{9}$ J. Mizuguchi, Z. Kristallogr. - New Cryst. Struct. 218, 141 (2003).

${ }^{10}$ M. Jost, A. Iqbal, and A. C. Rochat, U.S. Patent 4,585,878 (1986)

${ }^{11}$ J. Zambounis, Z. Hao, and A. Iqbal, European Patent 0673940 A1 (1995).

${ }^{12}$ J. Mizuguchi, Cryst. Res. Technol. 16, 695 (1981).

${ }^{13}$ G. M. Sheldrick, SHELXS86 (University of Göttingen, Germany, 1985).

${ }^{14}$ TEXSAN, Version 1.11, Molecular Structure Corporation (MSC), 9009 New Trails Drive, The Woodlands, TX 77381-5209, USA (2001).

${ }^{15}$ Quantum CAChe, Version 3.2, Fujitsu, Ltd., Kanagawa, Japan.

${ }^{16}$ J. Mizuguchi, A. Grubenmann, G. Wooden, and G. Rihs, Acta Crystallogr., Sect. B: Struct. Sci. 48, 696 (1992).

${ }^{17}$ J. Mizuguchi, A. Grubenmann, and G. Rihs, Acta Crystallogr., Sect. B: Struct. Sci. 49, 1056 (1993).

${ }^{18}$ B. di Bartolo, Optical Interactions in Solids (John Wiley \& Sons, Inc., New York, 1968). 